Research Article

\title{
The Large-Scale Synthesis of Vinyl-Functionalized Silicon Quantum Dot and Its Application in Miniemulsion Polymerization
}

\author{
Xuan-Dung Mai and Quang-Bac Hoang \\ Department of Chemistry, Hanoi Pedagogical University No. 2, 32 Nguyen Van Linh, Xuan Hoa, Phuc Yen, Vinh Phuc, Vietnam \\ Correspondence should be addressed to Xuan-Dung Mai; mdung.hpu2@gmail.com
}

Received 4 September 2016; Accepted 28 November 2016

Academic Editor: Miguel A. Garcia

Copyright ( 2016 X.-D. Mai and Q.-B. Hoang. This is an open access article distributed under the Creative Commons Attribution License, which permits unrestricted use, distribution, and reproduction in any medium, provided the original work is properly cited.

\begin{abstract}
Stable luminescence, size-tunability, and biocompatibility encourage the deployment of Cd-free NPs into diverse biological applications. Here we report one-pot synthesis of blue-emitting and polymerizable silicon quantum dots (Si QDs) from which water-soluble Si QDs embedded polystyrene nanoparticles (SiQD@PS NPs) were prepared using a miniemulsion polymerization approach. The hydrodynamic size of NPs was controlled by KOH to oleic acid molar ratio. Studies on the photoluminescence properties of SiQD@PS NPs in different conditions reveal that they exhibit two-photon luminescence property and high stability against $\mathrm{pH}$ and UV exposure. These NPs add new size regime to the Si QDs based luminescent makers for bioimaging and therapy applications.
\end{abstract}

\section{Introduction}

Advances in the synthesis and surface engineering of colloidal silicon quantum dots (Si QDs) [1-4] have promoted their deployment in many important applications including solar cells [5], light-emitting diodes [6], solution-process semiconductors [7], memory [8], and biological applications [9]. Though Si QDs may lag behind $\mathrm{Cd}$ or Pb based counterparts in the fields of optoelectronics, they have emerged as materials of choice for both in vitro and in vivo probes, from high-resolution imaging [3] to selective labeling of organelles [10], molecular coding [11], and tumor cell tracking [12] owing to their excellent biocompatibility, biodegradability $[13,14]$, photostability [3], and modest luminescence tunability $[15,16]$. For the examined potential of Si QDs to be widespread into these important applications, synthetic methods need further improvements in terms of scale-up, narrow size distribution, environmental amity, and costeffectiveness. A method developed by Hessel and coworkers can produce controllable and narrow size-distributed Si QDs with size-dependent luminescent properties through hightemperature thermal annealing hydrogen silsesquioxane in a reducing atmosphere $[2,17]$. However, this method requires the use of hazardous $\mathrm{HF}$ acid to liberate $\mathrm{SiO}_{2}$ matrix, the common drawback seen in many other synthetic protocols (e.g., laser pyrolysis, electrochemical or chemical etching, and plasma-assisted synthesis). Tilley and coworkers introduced a microemulsion approach to prepare hydrogen-terminated $\mathrm{Si}$ QDs via reducing silicon precursor (e.g., $\mathrm{SiCl}_{4}$ ) confined in inverse micelles of surfactant such as tetraoctylammonium bromide (TOAB) [18]. Since surface $\mathrm{Si}-\mathrm{H}$ bonds are easily oxidized when exposed to air atmosphere, the hydrogenterminated Si QDs are then reacted with various organic molecules with an end $\mathrm{C}=\mathrm{C}$ double bond functional group to perform either oil- or water-soluble Si QDs [1]. Although this solution-based synthetic protocol provides a versatile tool for designing the surfaces of Si QDs toward targeted applications $[4,8,19]$, only small amount of Si QDs could be obtained [20] with wasted Pt catalyst and TOAB. Wang et al. [21] and Cheng et al. [22] replaced TOAB with bipolar alkyl trichlorosilane, which self-assembled with $\mathrm{SiCl}_{4}$ in a micellar fashion followed by a $\mathrm{LiAlH}_{4}$ reduction step to perform alkyl-terminated Si QDs with relatively oxidized surfaces. The use of alkyl trichlorosilane as a triple substance, 
that is, surfactant, reagent, and alkyl capping precursor, simplifies the solution-based microemulsion synthetic protocol, which possibly enable mass production of cost-effective Si QDs. Unfortunately, the photostability of QDs, one of the important parameters for long-term luminescent probe applications, was not reported. It is worthy to note that the emission color of Si QDs is extremely sensitive to surface oxidation or functional groups $[4,15,16]$. Size-dependent red emission of hydrogen-terminated Si QDs changes into surface-assisted blue emission by exposing the QDs to mild oxidative environments [15]. We observed in a previous study that an additional yellow-emission band appeared when originally blue-emitting Si QDs got further oxidation in ambient conditions [4]. Even dodecyl-capped Si QDs also exhibit unstable red emission after storing in air for weeks (Figure S1 in Supplementary Material available online at http://dx.doi.org/10.1155/2016/2490235). These instabilities desire capping strategies to maintain the optical properties prior to the implementation of $\mathrm{Si}$ QDs into bioapplications. To this end, water-soluble Si QDs synthesized via a microwave-assisted protocol as proposed by Zhong et al. could be materials of examples [3]. Additionally, fluorescence intermittency or blinking commonly observed in single QD emission [23] and uninvited surface-related unhealthy reactions (e.g., formation of oxygen radicals [24]) need to be taken into account.

Herein, we adopted the microemulsion approach to synthesize large-scale vinyl-functionalized silicon quantum dots (V-Si QDs) using 7-octenyltrichlorosilane (OTS) as cocapping and stabilizing agent. Oil-soluble V-Si QDs were then subjected to a miniemulsion polymerization procedure with styrene monomer to perform water-soluble SiQD@PS NPs with the aid of in situ formed potassium oleate surfactant. Hydrodynamics size of the particles was controlled by varying the oleic acid to potassium hydroxide ratio. Polystyrene encapsulation enhanced the photostability of $\mathrm{Si}$ QDs while the luminescence exhibited insensitivity to $\mathrm{pH}$. The particles reported herein add new regime of biocompatible, photostable, and size-tunable luminescent probes for future biological applications.

\section{Materials and Method}

2.1. Synthesis of Vinyl-Functionalized Silicon Quantum Dot (VSi QDs). To $200 \mathrm{~mL}$ of anhydrous toluene (Aldrich), $0.8 \mathrm{~mL}$ (3.5 mmol) of OTS (98\%, Gelest) and $0.4 \mathrm{~mL}(3.5 \mathrm{mmol})$ of $\mathrm{SiCl}_{4}$ (99.998\%, Aldrich) were added with sonication for 30 minutes following vigorous stirring for 2 hour under $\mathrm{Ar}$ atmosphere to obtain microemulsion system of $\mathrm{SiCl}_{4}$ in OTS. $18 \mathrm{~mL}(36 \mathrm{mmol})$ of $\mathrm{LiAlH}_{4}$ solution (2 M in tetrahydrofuran, Aldrich) was then added dropwise and the mixture was further stirred for 2 hours to complete reduction of chlorosilane into silicon. The remaining $\mathrm{LiAlH}_{4}$ was quenched with $10 \mathrm{~g}$ (74 mmol) of $\mathrm{CuCl}_{2}$ (Aldrich), which was found to reduce uninvited oxidation of Si QDs as compared with methanol [19]. Next, the resultant mixture was dried using a rotary evaporator under reduced pressure. V-Si QDs were extracted by $50 \mathrm{~mL}$ of $\mathrm{n}$-hexane and filtered through $100 \mathrm{~nm}$ pore sized PTEE filters.
2.2. The Synthesis of Polystyrene NPs Embedding Si QDs (SiQD@PS). An oil solution consisting of $1 \mathrm{~g}$ ( $9.6 \mathrm{mmol})$ of freshly distillated styrene (Aldrich), $0.15 \mathrm{~g}$ of V-Si QDs, $0.8 \mathrm{~g}(2.8 \mathrm{mmol})$ of oleic acid (99\%, Aldrich), and $0.001 \mathrm{~g}$ of initiator $2,2^{\prime}$-azobis(2-methylpropionitrile) (AIBN, 99\%, Dejung Chemical) was prepared at freezing temperatures in an ice-bath. In a separate flask, a varied amount of $\mathrm{KOH}$ (5.6; 4.2; or $2.8 \mathrm{mmol}$ ) was dissolved in $20 \mathrm{~mL}$ of DI water. The oil phase was then transferred into the aqueous phase followed by 30 minutes of magnetic string and 15 minutes of ultrasonication to perform microemulsion system of oilin-water. During the mixing process, oleic acid in the oil phase reacted with $\mathrm{KOH}$ in the aqueous phase forming oleate, which acted as surfactant stabilizing the micelles. The mixture was heated up and maintained at $70^{\circ} \mathrm{C}$ for 12 hours followed by cooling down to room temperature to obtain SiQD@PS colloidal solution, which was then neutralized with $\mathrm{HCl}(1 \mathrm{M})$ for characterizations. The experimental procedures preparing V-Si QDs and SiQD@PS NPs are illustrated in Scheme 1.

2.3. Characterizations. Absorption spectra were obtained by using an S-3150 UV/Vis spectrometer (SCINCO, Korea). Photoluminescence and excitation spectra were performed on a Fluorolog-3 spectrometer (HORIBA Jobin Yvon, Inc., NJ) with a $2 \mathrm{~nm}$ slit width for both excitation and emission monochromators. Fourier transform infrared spectroscopy (FT-IR) was performed on a Spectrum 400 (PerkinElmer, MA) to obtain IR spectra of V-Si QDs and SiQD@PS. Highresolution transmission electron microscopy (HR-TEM) was performed with a TecnaiG2 F30 microscope (FEI, Hillsboro, Oregon) operated at $300 \mathrm{kV}$ in the bright-field mode. Hydrodynamic size and surface charge of SiQD@PS NPs were analyzed with a surface zeta potential and particle size analyzer (Otsuka Electronics, ELS-8000).

\section{Results and Discussion}

Simple, cost-effective, and large-scale syntheses of Si QDs are of importance to implement these novel nanosized semiconductors into real applications. In a previous study [8], we simply reduced $\mathrm{SiCl}_{4}$ ultrasonicated in toluene to hydrogen-terminated $\mathrm{Si}$ QDs, which were then refluxed with styrene monomer to perform a nanocomposite having charge trapping ability. The lack of surfactant such as TOAB simplified the process, but it resulted in relative broad size distribution. Herein, as cartooned in Scheme 1(a), we added OTS having both a polar $-\mathrm{SiCl}_{3}$ head group and a nonpolar 7octenyl tail, hence acting as a surfactant, with a hope to focus the size of resultant Si QDs in a similar manner to previous reports $[21,22]$.

As expected, TEM images shown in Figure 1(a) revealed that the size distribution of V-Si QDs is monodisperse with an average diameter of $3.5 \pm 1 \mathrm{~nm}$, which is comparable with the Bohr radius of bulk silicon $(4.3 \mathrm{~nm})$. Moreover, HR-TEM image (Figure 1(a) inset) indicated that V-Si QDs are crystalline. In addition to size focusing, the use of OTS instead of TOAB simplified purification processes and avoided a surface capping step that is usually conducted to acquire air-stable colloidal Si QDs. As a result, a much larger 

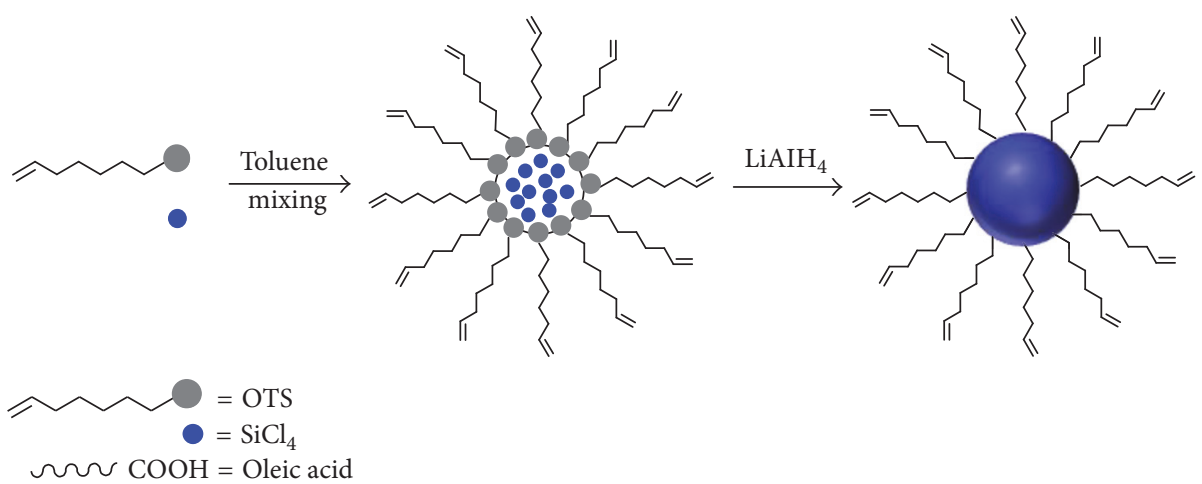

(a)

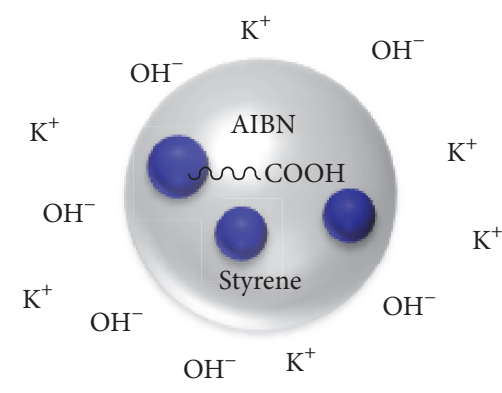

Oil/water emulsion
$\mathrm{K}^{+}$

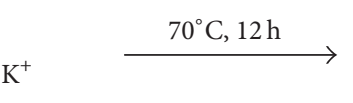

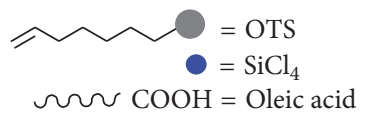

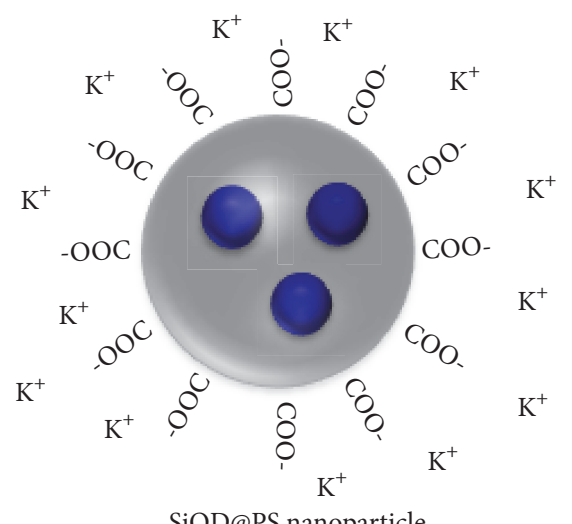

SiQD@PS nanoparticle

(b)

Scheme 1: Surfactant-free synthesis of vinyl-terminated Si QDs (a) and miniemulsion polymerization synthesis of Si QDs embedded polystyrene NPs (b).

amount of V-Si QDs could be obtained without consuming costly Pt catalyst or wasting TOAB. For a typical synthetic bath, about $0.5(\mathrm{~g})$ of $\mathrm{V}$-Si QDs was obtained, which was approximately 3 times greater than the conventional synthetic protocol using TOAB as stabilizer [4].

Optical properties of V-Si QDs dispersed in n-hexane are summarized in Figure 1(b). UV-vis absorption spectrum (blue line) exhibits a sharp rise in absorption in high-energy region staring at $290 \mathrm{~nm}(4.3 \mathrm{eV})$ and a long low-absorption tail from $290 \mathrm{~nm}$ to $430 \mathrm{~nm}(2.9 \mathrm{eV})$, which can be assigned to direct and indirect transitions in the crystalline Si QDs, respectively [25]. The energies are, respectively, higher than the direct bandgap $(3.4 \mathrm{eV})$ at $\Gamma$ or the indirect bandgap $(1.1 \mathrm{eV})$ of bulk Si being due to quantum confinement effects [25]. The dependence of photoluminescence (PL) spectra of $\mathrm{V}$-Si QDs dispersed in n-hexane on excitation wavelength shown in Figure 1(b) reveals that the highest emission efficiency was achieved with an excitation wavelength at ca. 340$360 \mathrm{~nm}$. The absolute emission quantum yield, conducted on an integration sphere, was $12 \%$ at an excitation wavelength of $350 \mathrm{~nm}$. Additionally, PL spectra exhibit vibrational features and asymmetric shape with higher contribution of longwavelength side. These phenomena are usually observed on solution-synthetic Si QDs with rich surface states originated from oxidation [4]. Surface $\mathrm{Si}-\mathrm{O}$ bonds add new electronic states to crystalline Si QD core, some of which align within the bandgap narrowing the optical gap of Si QD, called surface states. Moreover, the oxidation states may localize excited carriers leading to enhanced electron-phonon coupling giving rise to the observed vibrational features in $\mathrm{PL}$ spectra [26].

The oxidation was further confirmed by an absorption band centered $1068 \mathrm{~cm}^{-1}$ in the FT-IR spectrum of V-Si QDs shown in Figure 2(a), which was attributed to oxidized $\mathrm{SiO}_{x}$ component. Additionally, characteristic IR peaks of 1-octene including $3078 \mathrm{~cm}^{-1}$ (=C-H stretching), $1643 \mathrm{~cm}^{-1}$ (-C=Cstretching), $991 \mathrm{~cm}^{-1}$ and $910 \mathrm{~cm}^{-1}$ (=C-H bending), and $2852 \mathrm{~cm}^{-1}$ and $2821 \mathrm{~cm}^{-1}$ (-C-H stretching) are also visible in the FT-IR spectrum of V-Si QDs, thus confirming the existence of 7-octenyl groups on Si QDs surfaces. In summary, by using OTS instead of conventional TOAB surfactant to simplify the microemulsion synthetic protocol, we were able 


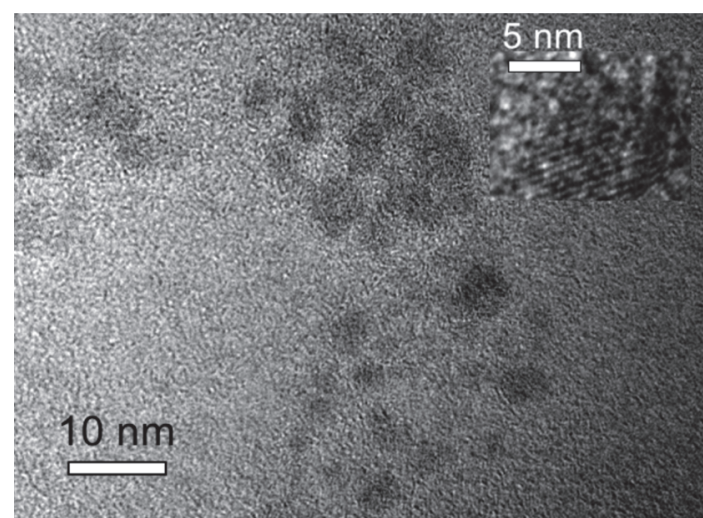

(a)

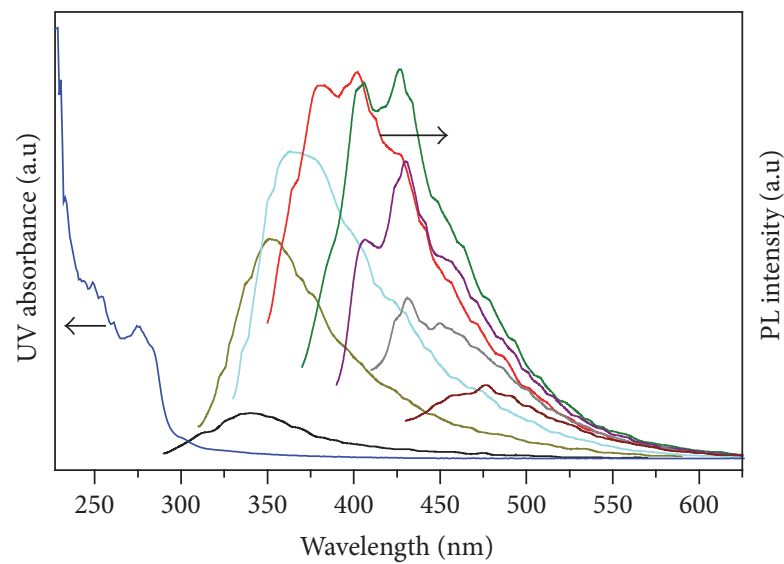

PL

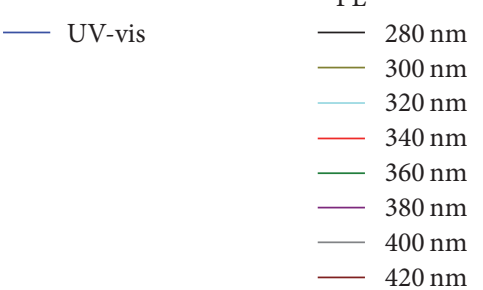

(b)

FIGURE 1: (a) TEM images and (b) optical properties of vinylterminated Si QDs. In (b) UV-vis absorbance and photoluminescent spectra (PL) at different excitation wavelengths were measured at room temperature in $n$-hexane solutions.

to synthesize a large scale of colloidal Si QDs with a good emission quantum efficiency of $12 \%$. The surface of V-Si QDs was partially oxidized and bearing vinyl functional groups, which could be converted into polar groups (e.g., $-\mathrm{NH}_{2}$, $\mathrm{COOH}$, and $-\mathrm{SO}_{3}[22]$ ) performing small water-soluble $\mathrm{Si}$ QDs or be polymerized with other monomers such as styrene to synthesize nanocomposites [8].

Cd-free photoluminescence nanoparticles have emerged as potential materials for bioimaging applications in which hydrodynamics sizes, surface chemistry, and photostability are important parameters determining whether the particles are useful $[10,27]$. Yet, water-soluble colloidal Si QDs and surfactant-encapsulated Si QDs have been studied for diverse bioimaging applications. The colloidal Si QDs with

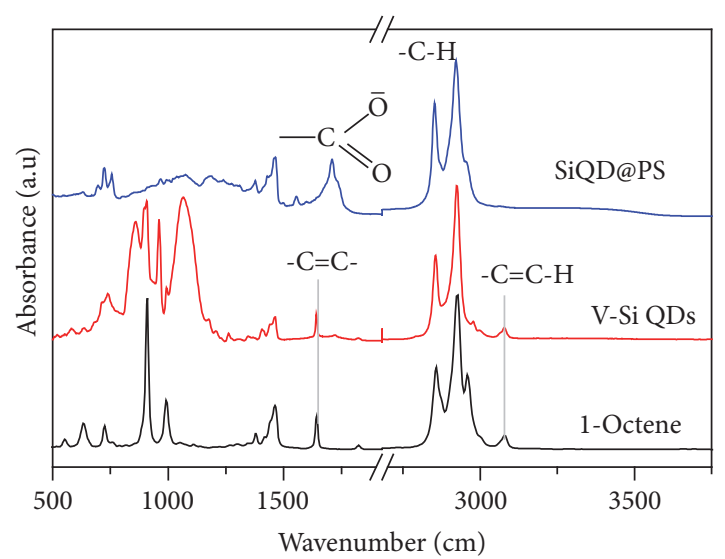

(a)

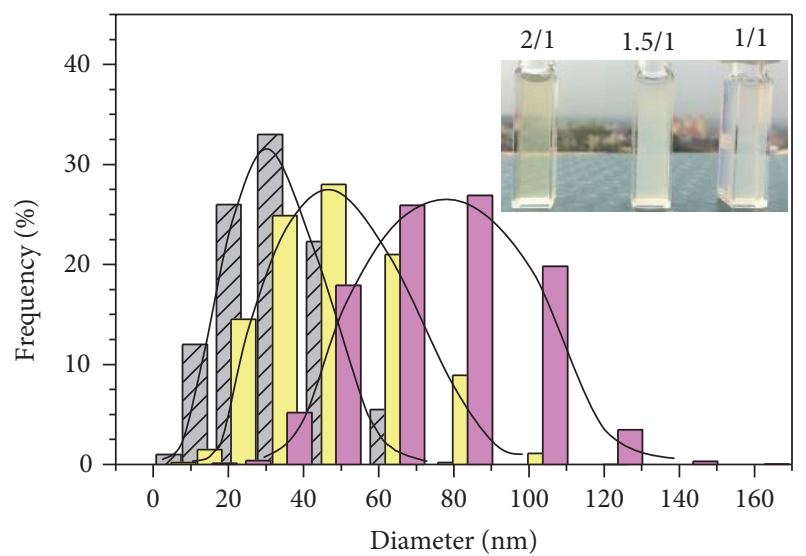

$\mathrm{KOH} / \mathrm{OA}=$

पIत $2 / 1$

$\square 1.5 / 1$

$\square 1 / 1$

(b)

FIGURE 2: (a) FT-IR spectra of SiQD@PS nanoparticles, V-Si QDs, and 1-octene. (b) Size distribution of SiQD@PS nanoparticles obtained when varying the $\mathrm{KOH}$ to oleic acid (OA) molar ratio. Inset: pictures of SiQD@PS nanoparticles dispersed in water under daylight showing different turbidity.

a hydrodynamic size in order of $10 \mathrm{~nm}$ may have fast distribution and urine clearance but they commonly exhibit luminescence blinking properties [23]. Surfactant-stabilized aggregates of QDs contain some QDs so as to give continuous emission being suitable for tracking applications [28]; their size is hardly controlled because it depends on the chemical structure of surfactant. Herein we encapsulated V-Si QDs into polystyrene NPs to not only prevent V-Si QDs from further oxidation, hence preserving their luminescence, but also produce biocompatible NPs whose hydrodynamic sizes could be controlled easily. As cartooned in Scheme 1, an oil phase containing V-Si QDs, styrene comonomer, and AIBN radical initiator was stabilized in water by in situ formed potassium oleate surfactant. Upon heating, the decomposition of AIBN initiated copolymerization between V-Si QDs and styrene performing hard sphere polystyrene nanoparticles, named as 


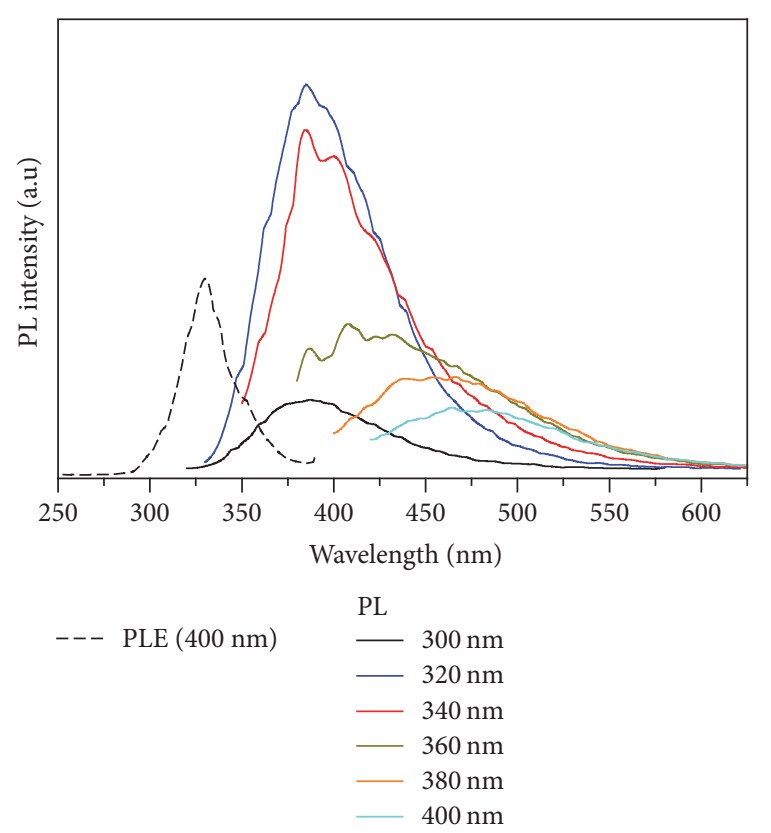

(a)

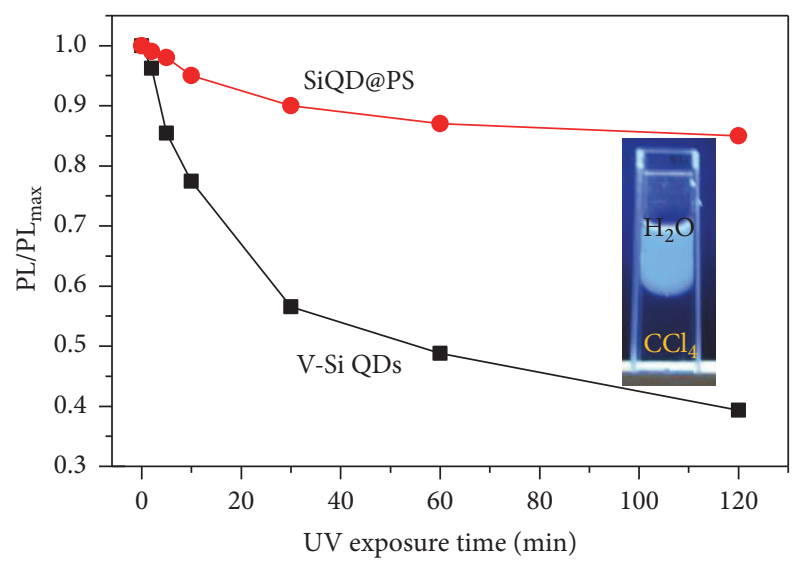

(b)

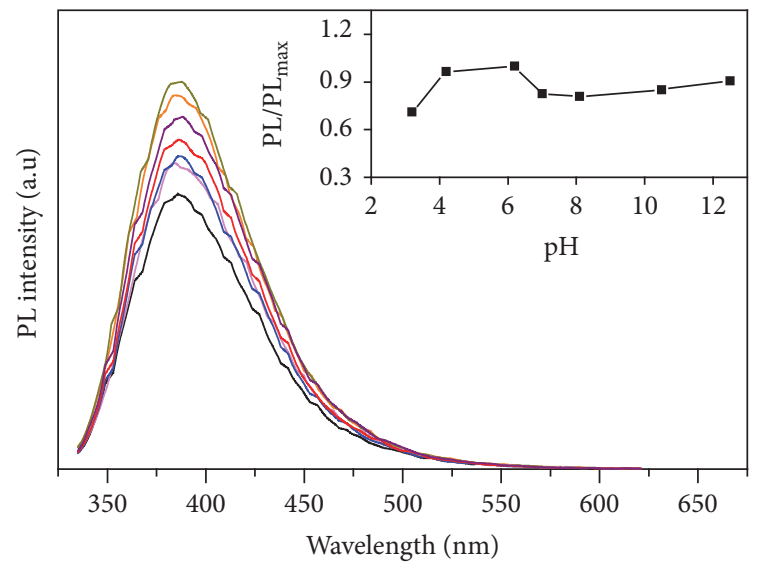

$\mathrm{pH}=$

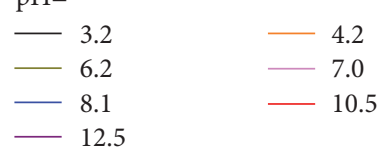

(c)

FIGURE 3: Continued.

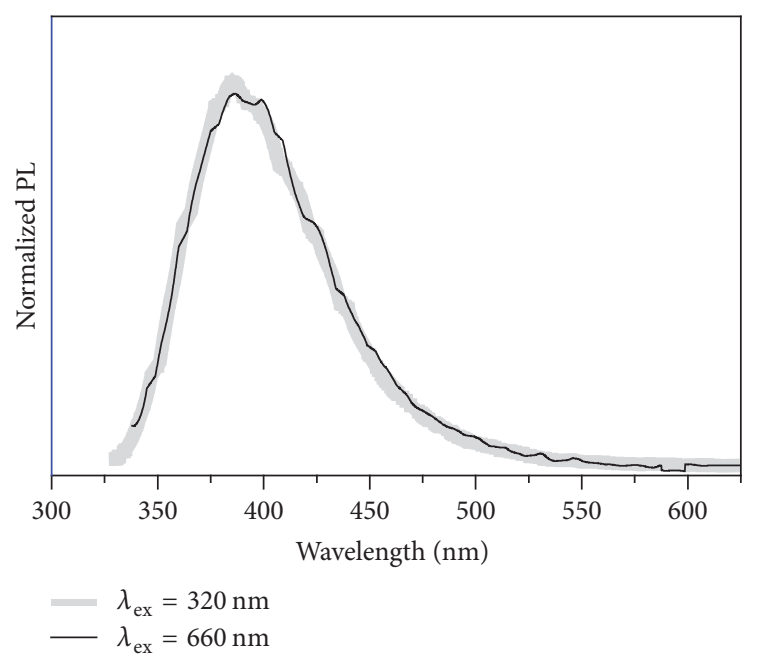

(d)

FIgURE 3: Optical properties of SiQD@PS nanoparticles prepared using $\mathrm{KOH} / \mathrm{OA}=2$. (a) Photoluminescence spectra (PL) obtained at different excitation wavelengths and photoluminescence excitation (PLE) spectrum conducted at $400 \mathrm{~nm}$. (b) Photostability of SiQD@PS nanoparticles in comparison with V-Si QDs. Inset: picture of SiQD@PS nanoparticles dispersed in water (upper layer) after two hours of excitation with an UV lamp. (c) $\mathrm{pH}$ stability of photoluminescence. The PL spectra were conducted with an excitation wavelength of $320 \mathrm{~nm}$. pH was varied with $\mathrm{KOH}$ or $\mathrm{HCl}$ $1 \mathrm{M}$ solutions. Inset: variation of PL intensity at $386 \mathrm{~nm}$ with $\mathrm{pH}$. (d) Normalized PL spectra obtained with an excitation wavelength at 320 or $640 \mathrm{~nm}$ showing two-photon luminescence property of SiQD@PS NPs.

SiQD@PS NPs. The size of the oil phase, hence the diameter of SiQD@PS NPs, was governed by some parameters such as oil/surfactant/water mass ratio and ionic strength of the water phase.

FT-IR spectrum of SiQD@PS NPs synthesized according to Scheme $1(\mathrm{~b})$ is shown in Figure 2(a). A peak at $1708 \mathrm{~cm}^{-1}$ is attributed to $\mathrm{C}=\mathrm{O}$ stretching of surface carboxylate groups that enable water-solubility of SiQD@PS NPs (inset in Figure 2(b)). The hydrodynamic size distribution of SiQD@PS NPs measured by DLS technique is shown in Figure 2(b). All samples were monodisperse; their average hydrodynamic diameter increased from 34 to 48 and then to $75 \mathrm{~nm}$ when decreasing $\mathrm{KOH}$ to oleic acid molar ratio from $2: 1$ to $1.5: 1$ and then to $1: 1$, respectively. These sizes were relatively larger than the diameters determined from FE-SEM images; for example, the diameter of the smallestsize samples was $25 \mathrm{~nm}$ (Figure S2). This difference can be attributed to solvated carboxylate groups on the surfaces of SiQD@PS nanoparticles. The increase in particle size is visible in the inset in Figure 2(b) that shows decreasing transparence with decreasing $\mathrm{KOH} /$ oleic acid molar ratio.

Optical properties of SiQD@PS NPs prepared using $\mathrm{KOH} / \mathrm{OA}=2$ are summarized in Figure 3. PL spectra (Figure 3(a)) of SiQD@PS NPs dispersed in water were slightly 
blue-shifted of ca. $15 \mathrm{~nm}$ and less asymmetric compared with those of V-Si QDs. These behaviors can be attributed to surface polystyrene encapsulation that prevents V-Si QDs from uninvited oxidation and suppresses some surface-related emission centers on V-Si QDs. Full photoluminescence excitation spectrum (PLE) observed at $400 \mathrm{~nm}$ reveals that the emission is maximized at $330 \mathrm{~nm}$ excitation. Excitation with wavelengths shorter than $290 \mathrm{~nm}$ was negligible due to light scattering. The scattering effects were also the main reason that inhibited a direct comparison of the relative PL quantum yields between V-SiQDs and size-different SiQD@PS NPs.

For actual applications, the photoluminescence of nanoprobes should be stable with excitation and environment. The photostability of SiQD@PS NPs was evaluated by measuring their PL spectra with the same conditions after placing the solution on a $6 \mathrm{~W}$-UV lamp that emitted at $365 \mathrm{~nm}$ with different periods; the results are shown in Figure 3(b) in comparison with V-Si QDs. Obviously, while PL of V-Si QDs decreased quickly by UV exposure, SiQD@PS nanoparticles maintained their luminescence by more than $85 \%$ after 2 hours of excitation. In addition to enhanced photostability, the emission efficiency of SiQD@PS NPs slightly fluctuated with $\mathrm{pH}$, Figure 3(c). It should be noted that, with pH lower than 3, SiQD@PS NPs tended to aggregate. Probably, under acidic conditions, the surface carboxylate groups converted into carboxyl groups giving zero-charged particles that are unstable colloids. Finally, bioapplicability of SiQD@PS NPs was corroborated by their two-photon luminescence ability; the property endows excitation nanoprobes with long-wavelength light that is less scattered by tissues [28]. As shown in Figure 3(d), normalized PL spectra of SiQD@PS NPs were identical with either excitation wavelength at $320 \mathrm{~nm}$ or $640 \mathrm{~nm}$.

\section{Conclusions}

In this report, we have demonstrated a new scalable synthetic protocol using 7-octenyltrichlorosilane instead of conventional TOAB surfactant to produce oil-soluble, strongly luminescent Si QDs having vinyl functional groups on the surfaces. The 7-octenyl groups were not able to prevent fully Si QDs from surface oxidation that facilitated surface-related emission. Encapsulation of Si QDs into watersoluble polystyrene NPs via a miniemulsion polymerization technique was found to enhance the photostability of luminescence, which also showed fairly stable $\mathrm{pH}$ and exhibited two-photon excitation capability. Additionally, the hydrodynamic size of NPs was easily controlled, ranging from 34 to $75 \mathrm{~nm}$, by varying $\mathrm{KOH}$ to oleic acid ratio. The luminescent NPs demonstrated herein with novel properties, such as biocompatible, size-tunable, and advanced photoluminescence properties promise potential applications as nanoprobes.

\section{Competing Interests}

The authors declare that they have no competing interests.

\section{Acknowledgments}

This research was supported by Hanoi Pedagogical University No. 2 under Grant no. C.2015-18-3.

\section{References}

[1] R. D. Tilley and K. Yamamoto, "The microemulsion synthesis of hydrophobic and hydrophilic silicon nanocrystals," Advanced Materials, vol. 18, no. 15, pp. 2053-2056, 2006.

[2] C. M. Hessel, D. Reid, M. G. Panthani et al., "Synthesis of ligand-stabilized silicon nanocrystals with size-dependent photoluminescence spanning visible to near-infrared wavelengths," Chemistry of Materials, vol. 24, no. 2, pp. 393-401, 2012.

[3] Y. Zhong, F. Peng, F. Bao et al., "Large-scale aqueous synthesis of fluorescent and biocompatible silicon nanoparticles and their use as highly photostable biological probes," Journal of the American Chemical Society, vol. 135, no. 22, pp. 8350-8356, 2013.

[4] M. X. Dung, D. D. Tung, S. Jeong, and H.-D. Jeong, “Tuning optical properties of $\mathrm{Si}$ quantum dots by $\pi$-conjugated capping molecules," Chemistry, vol. 8, no. 3, pp. 653-664, 2013.

[5] C.-Y. Liu and U. R. Kortshagen, "A Silicon nanocrystal schottky junction solar cell produced from colloidal silicon nanocrystals," Nanoscale Research Letters, vol. 5, no. 8, pp. 1253-1256, 2010.

[6] D. P. Puzzo, E. J. Henderson, M. G. Helander, Z. Wang, G. A. Ozin, and Z. Lu, "Visible colloidal nanocrystal silicon lightemitting diode," Nano Letters, vol. 11, no. 4, pp. 1585-1590, 2011.

[7] R. Gresback, N. J. Kramer, Y. Ding, T. Chen, U. R. Kortshagen, and T. Nozaki, "Controlled doping of silicon nanocrystals investigated by solution-processed field effect transistors," ACS Nano, vol. 8, no. 6, pp. 5650-5656, 2014.

[8] M. X. Dung, J.-K. Choi, and H.-D. Jeong, "Newly synthesized silicon quantum dot-polystyrene nanocomposite having thermally robust positive charge trapping," ACS Applied Materials and Interfaces, vol. 5, no. 7, pp. 2400-2409, 2013.

[9] O. S. Wolfbeis, "An overview of nanoparticles commonly used in fluorescent bioimaging," Chemical Society Reviews, vol. 44, no. 14, pp. 4743-4768, 2015.

[10] S. Ohta, P. Shen, S. Inasawa, and Y. Yamaguchi, "Size- and surface chemistry-dependent intracellular localization of luminescent silicon quantum dot aggregates," Journal of Materials Chemistry, vol. 22, no. 21, pp. 10631-10638, 2012.

[11] F. Erogbogbo, K.-T. Yong, R. Hu et al., "Biocompatible magnetofluorescent probes: luminescent silicon quantum dots coupled with superparamagnetic iron(III) oxide," ACS Nano, vol. 4, no. 9, pp. 5131-5138, 2010.

[12] X. Ji, F. Peng, Y. Zhong et al., "Highly fluorescent, photostable, and ultrasmall silicon drug nanocarriers for long-term tumor cell tracking and in-vivo cancer therapy," Advanced Materials, vol. 27, no. 6, pp. 1029-1034, 2015.

[13] J.-H. Park, L. Gu, G. Von Maltzahn, E. Ruoslahti, S. N. Bhatia, and M. J. Sailor, "Biodegradable luminescent porous silicon nanoparticles for in vivo applications," Nature Materials, vol. 8, no. 4, pp. 331-336, 2009.

[14] J. Liu, F. Erogbogbo, K.-T. Yong et al., "Assessing clinical prospects of silicon quantum dots: studies in mice and monkeys," ACS Nano, vol. 7, no. 8, pp. 7303-7310, 2013.

[15] M. Dasog, Z. Yang, S. Regli et al., "Chemical insight into the origin of red and blue photoluminescence arising from freestanding silicon nanocrystals," ACS Nano, vol. 7, no. 3, pp. 26762685, 2013. 
[16] M. Dasog, G. B. De Los Reyes, L. V. Titova, F. A. Hegmann, and J. G. C. Veinot, "Size vs surface: tuning the photoluminescence of freestanding silicon nanocrystals across the visible spectrum via surface groups," ACS Nano, vol. 8, no. 9, pp. 9636-9648, 2014.

[17] C. M. Hessel, E. J. Henderson, and J. G. C. Veinot, "Hydrogen silsesquioxane: a molecular precursor for nanocrystalline $\mathrm{Si}$ $\mathrm{SiO}_{2}$ composites and freestanding hydride-surface-terminated silicon nanoparticles," Chemistry of Materials, vol. 18, no. 26, pp. 6139-6146, 2006.

[18] R. D. Tilley, J. H. Warner, K. Yamamoto, I. Matsui, and H. Fujimori, "Micro-emulsion synthesis of monodisperse surface stabilized silicon nanocrystals," Chemical Communications, no. 14, pp. 1833-1835, 2005.

[19] M. X. Dung and H.-D. Jeong, "Synthesis of styryl-terminated silicon quantum dots: reconsidering the use of methanol," Bulletin of the Korean Chemical Society, vol. 33, no. 12, pp. 41854187, 2012.

[20] M. Rosso-Vasic, E. Spruijt, B. Van Lagen, L. De Cola, and H. Zuilhof, "Alkyl-functionalized oxide-free silicon nanoparticles: synthesis and optical properties," Small, vol. 4, pp. 1835-1841, 2008.

[21] J. Wang, S. Sun, F. Peng, L. Cao, and L. Sun, "Efficient one-pot synthesis of highly photoluminescent alkyl-functionalised silicon nanocrystals," Chemical Communications, vol. 47, no. 17, pp. 4941-4943, 2011.

[22] X. Cheng, R. Gondosiswanto, S. Ciampi, P. J. Reece, and J. J. Gooding, "One-pot synthesis of colloidal silicon quantum dots and surface functionalization via thiol-ene click chemistry," Chemical Communications, vol. 48, no. 97, pp. 11874-11876, 2012.

[23] B. Bruhn, J. Valenta, F. Sangghaleh, and J. Linnros, "Blinking statistics of silicon quantum dots," Nano Letters, vol. 11, no. 12, pp. 5574-5580, 2011.

[24] K. Fujioka, M. Hiruoka, K. Sato et al., "Luminescent passiveoxidized silicon quantum dots as biological staining labels and their cytotoxicity effects at high concentration," Nanotechnology, vol. 19, no. 41, Article ID 415102, 2008.

[25] J. P. Wilcoxon, G. A. Samara, and P. N. Provencio, "Optical and electronic properties of $\mathrm{Si}$ nanoclusters synthesized in inverse micelles," Physical Review B, vol. 60, no. 4, pp. 2704-2714, 1999.

[26] J. Martin, F. Cichos, F. Huisken, and C. Von Borczyskowski, "Electron-phonon coupling and localization of excitons in single silicon nanocrystals," Nano Letters, vol. 8, no. 2, pp. 656660, 2008.

[27] G. Ruan and J. O. Winter, "Alternating-color quantum dot nanocomposites for particle tracking," Nano Letters, vol. 11, no. 3, pp. 941-945, 2011.

[28] F. Erogbogbo, C.-A. Tien, C.-W. Chang, P. Adjei-Baffour, W.C. Law, and M. Swihart, "Biodegradable luminescent silicon quantum dots for two photon imaging applications," Nature Precedings, 2012. 

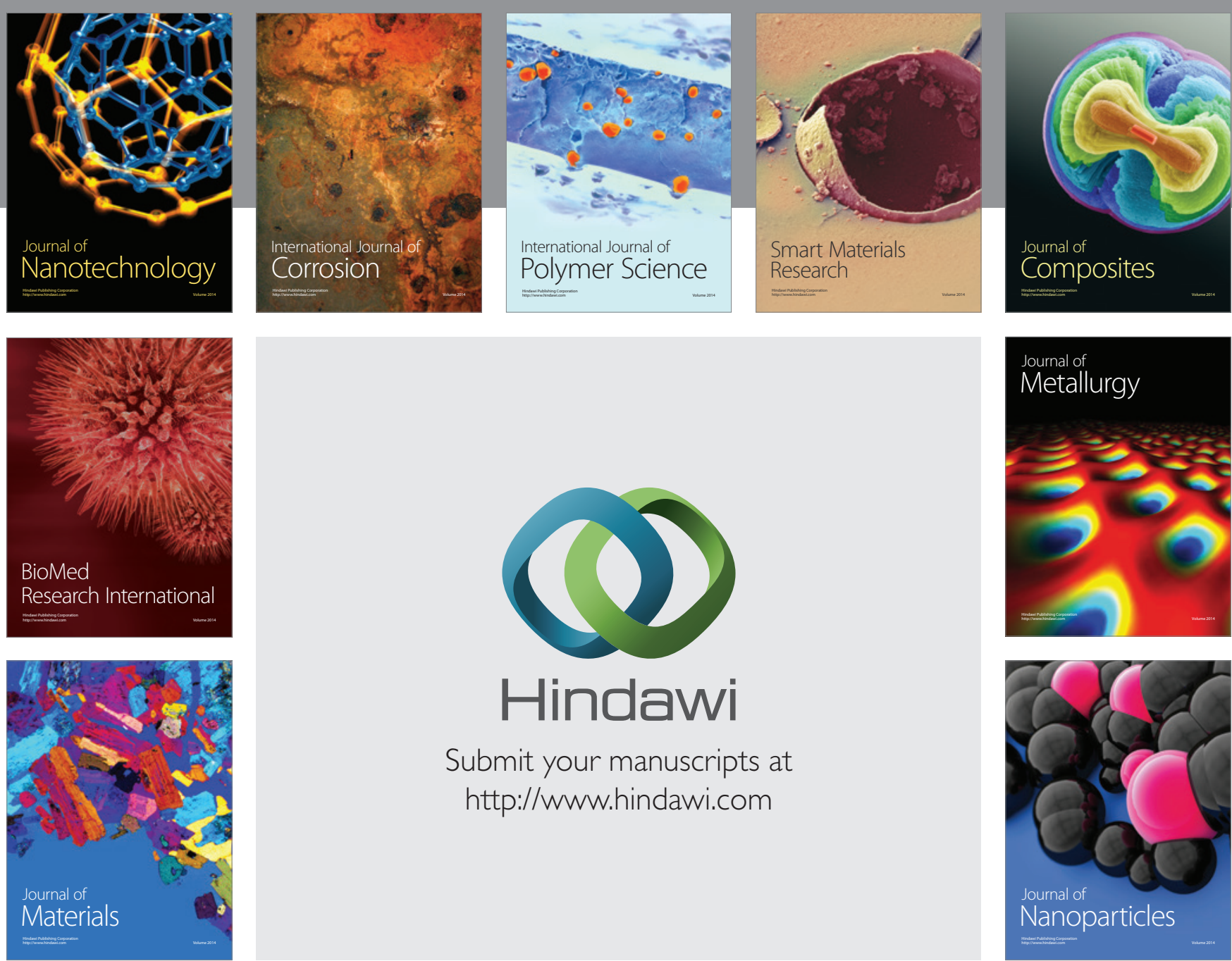

\section{Hindawi}

Submit your manuscripts at

http://www.hindawi.com

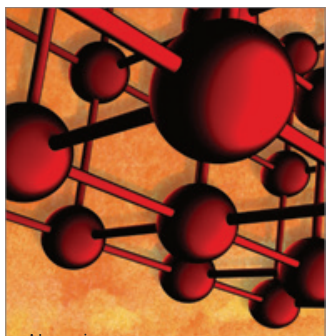

Materials Science and Engineering
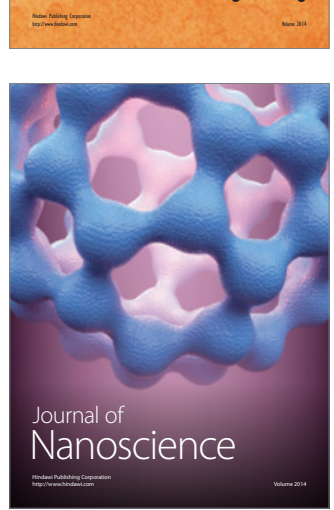
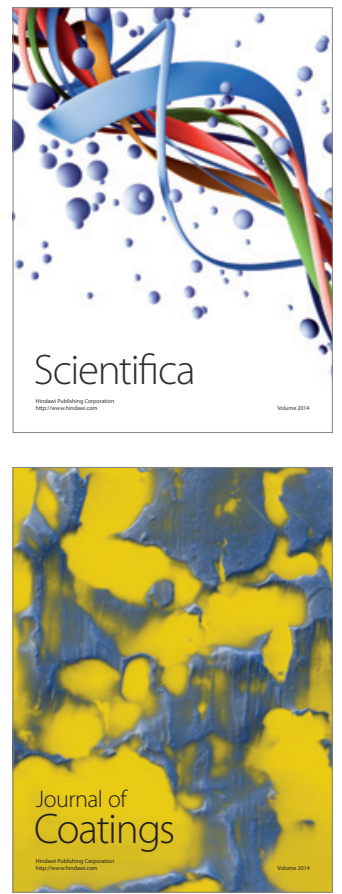
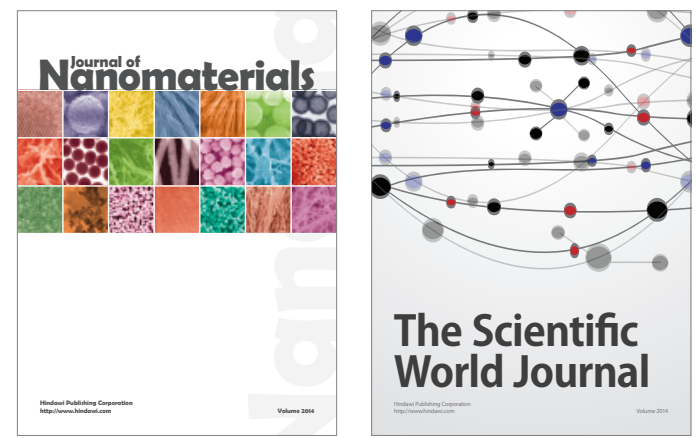

The Scientific World Journal
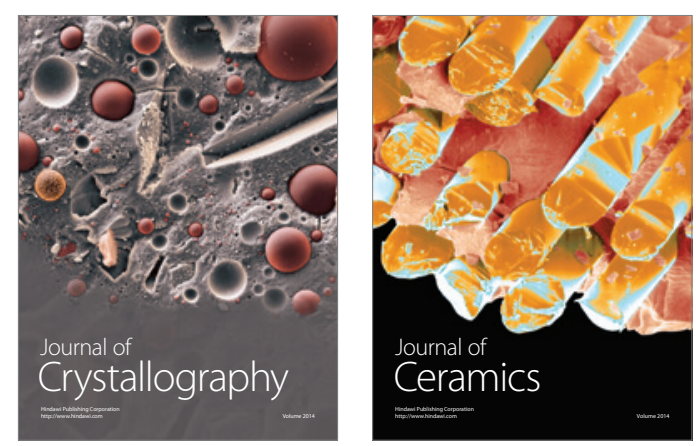
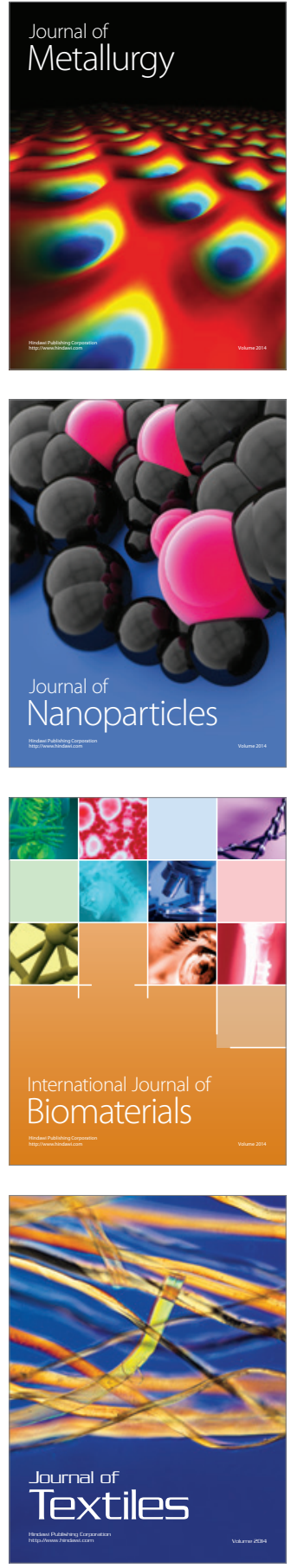